\title{
Narrow-Line AGNs: confirming the relationship between metallicity and accretion rate
}

\section{M. Neri-Larios*}

Departamento de Astronomía, Universidad de Guanajuato, 36000, Guanajuato, Mexico

E-mail: danieldastro.ugto.mx

\section{R. Coziol ${ }^{1}$, J.P. Torres-Papaqui ${ }^{1}$, H. Andernach ${ }^{1}$, J.M. Islas-Islas ${ }^{1}$, I. Plauchu-Frayn ${ }^{2}$,} and R.A. Ortega-Minakata ${ }^{1}$

1 Departamento de Astronomía, Universidad de Guanajuato, 36000, Guanajuato, Mexico

2 Instituto de Astrofísica de Andalucía (CSIC), E-18008, Granada, Spain

E-mail: rcozioldastro.ugto.mx, papaquidastro.ugto.mx,

heinzeastro.ugto.mx, jmislaseastro.ugto.mx, ilse@iaa.es,

renedastro.ugto.mx

We have selected a sample of 292 SDSS Narrow-Emission-Line galaxies (NELGs) known to have formed and evolved in relative isolation to study the nature and origin of the AGN phenomenon. The galaxies in our sample have line fluxes with $\mathrm{S} / \mathrm{N}>3$ and were separated using a standard diagnostic diagram into Star Forming Galaxies (SFGs; 36.0\%), Transition type Objects (TOs; 28.4\%) and Narrow-Line AGNs (NLAGNs; 35.6\%). Having found a strong correlation between the bulge mass and the NLAGN phenomenon, we have applied the same relation as for the BroadLine AGNs (BLAGNs) to estimate their black hole (BH) masses. The BH in the NLAGNs are 2 to 3 orders lower in mass than the BHs found in BLAGNs, but are comparable to those observed in Narrow-Line Seyfert 1 (NLS1), although none of our objects can be classified as such. To determine the metallicities, $[\mathrm{O} / \mathrm{H}]$, of the NLAGNs we calibrated the standard diagnostic diagram $[\mathrm{OIII}] / \mathrm{H} \beta$ vs. $[\mathrm{NII}] / \mathrm{H} \alpha$ using similar relation as for the SFGs, which reproduce the values obtained with CLOUDY simulations developed for Bennert et al. [3]. For some individual objects we compared our line ratios with other CLOUDY similations by different authors. This suggests we achieve a typical uncertainty of 0.2 dex on $[\mathrm{O} / \mathrm{H}]$, increasing to 0.3-0.5 in the Seyfert 2 (S2). This calibration suggests the metallicities of the NLAGNs are subsolar, varying between 1 and 0.3 $Z_{\odot}$. We find two statistically significant positive correlations: for $[\mathrm{O} / \mathrm{H}]$ with the $\mathrm{BH}$ mass and for $[\mathrm{O} / \mathrm{H}]$ with the luminosity at $5100 \AA, \lambda L(5100 \AA)$. No correlation is found between $[\mathrm{O} / \mathrm{H}]$ and the accretion rate, $L_{b o l} / L_{E d d}$. However, comparisons with the BLAGNs suggest the NLAGNs extend the metallicity-accretion rate relationship [24] to the low metallicity regime. Although the NLS1 have similar BH masses as the NLAGNs they show higher accretion rates, which is consistent with their higher metallicities.

Narrow-Line Seyfert 1 Galaxies and their place in the Universe - NLS1,

April 04-06, 2011

Milan Italy

* Speaker. 


\section{Introduction}

Spectroscopic surveys like the Sloan Digital Sky Survey (SDSS), have revealed that a very high number of galaxies in the nearby universe show narrow emission lines in their nucleus. Using spectroscopic diagnostic diagrams comparing various line ratios, different classification schemes were proposed to distinguish between the possible sources of excitation of the gas in these galaxies ([2]; [28]; [15]; [13]). These classification schemes suggest there are two main sources of ionization: thermal sources, which are related to star forming activity, and non thermal sources that are related to the possible accretion of matter by a massive black hole, the so-called Active Galactic Nuclei (AGNs).

Although most of the emission line galaxies in SDSS are ionized by stars, recent studies (e.g, [20]; [27]) suggest that the actual number of galaxies with an accreting black hole may equal that of the star forming galaxies. However, the evidence is obscured by the large variation of characteristics observed in AGNs. For example, in the so-called Seyfert 1 (S1) galaxies, where we distinguish broad emission line components akin to what is observed in quasars ([21]; [29]; [18]), the accretion of matter onto a supermassive BH seems obvious. But the situation is much more complicated with narrow-line AGNs (NLAGNs) where no broad emission lines are observed. We usually distinguish two kinds of NLAGNs: the high-ionization Seyfert 2 (S2) and the lowionization LINER, which stands for Low Ionization Nuclear Emission-line Region ([10]; [5]; [14]). Another case which is particularly intriguing is the narrow-line Seyfert 1 (NLS1), which seems to show the same high level of activity as the S1 but with narrower emission lines ([17]; [16]).

Understanding what is the nature of the differences between the various NLAGNs is an important matter and the subject of intense research activity. According to the unification model for AGNs, the difference between BLAGNs and NLAGNs is solely a question of geometry and orientation with respect to the line of sight: all these galaxies have a $\mathrm{BH}$ at their center, but in the NLAGNs the line of sight to the region producing the broad emission lines is obscured by a torus of matter and dust encircling the BH. However, the prevalent model to explain the NLS1 seems to suggest they have smaller mass BHs than the S1, and, like quasars, accrete matter near the Eddington limit [23]. But, what happens then for the S2 and LINER? Here we use a new sample of isolated galaxies classified as NLAGNs to study this problem.

\section{Description of the sample and determination of activity type}

By comparing the position of galaxies in the 2MASS-selected Isolated Galaxies Catalog (2MIG; [11]) with those in the Sloan Digital Sky Survey Data Release 7 (SDSS DR7, [1]) we have retrieved the spectra for 292 narrow emission line galaxies, with signal-to-noise ratios $\mathrm{S} / \mathrm{N} \geq 3.0$ for [OIII] $\lambda 5007, \mathrm{H} \beta$, [NII] $\lambda 6584$ and $\mathrm{H} \alpha$. The spectra were subsequently corrected for Galactic extinction, shifted to their rest frame, re-sampled to $\Delta \lambda=1 \AA$ between 3400 and $8900 \AA$, and processed using the spectral synthesis code STARLIGHT [4]. Using STARLIGHT the contribution of the underlying stellar population was effectively eliminated, allowing a high precision measure of the fluxes of the different emission lines observed. Through the template fitting technique, we also obtained an estimate of the stellar velocity dispersion in the bulge of the galaxies. 


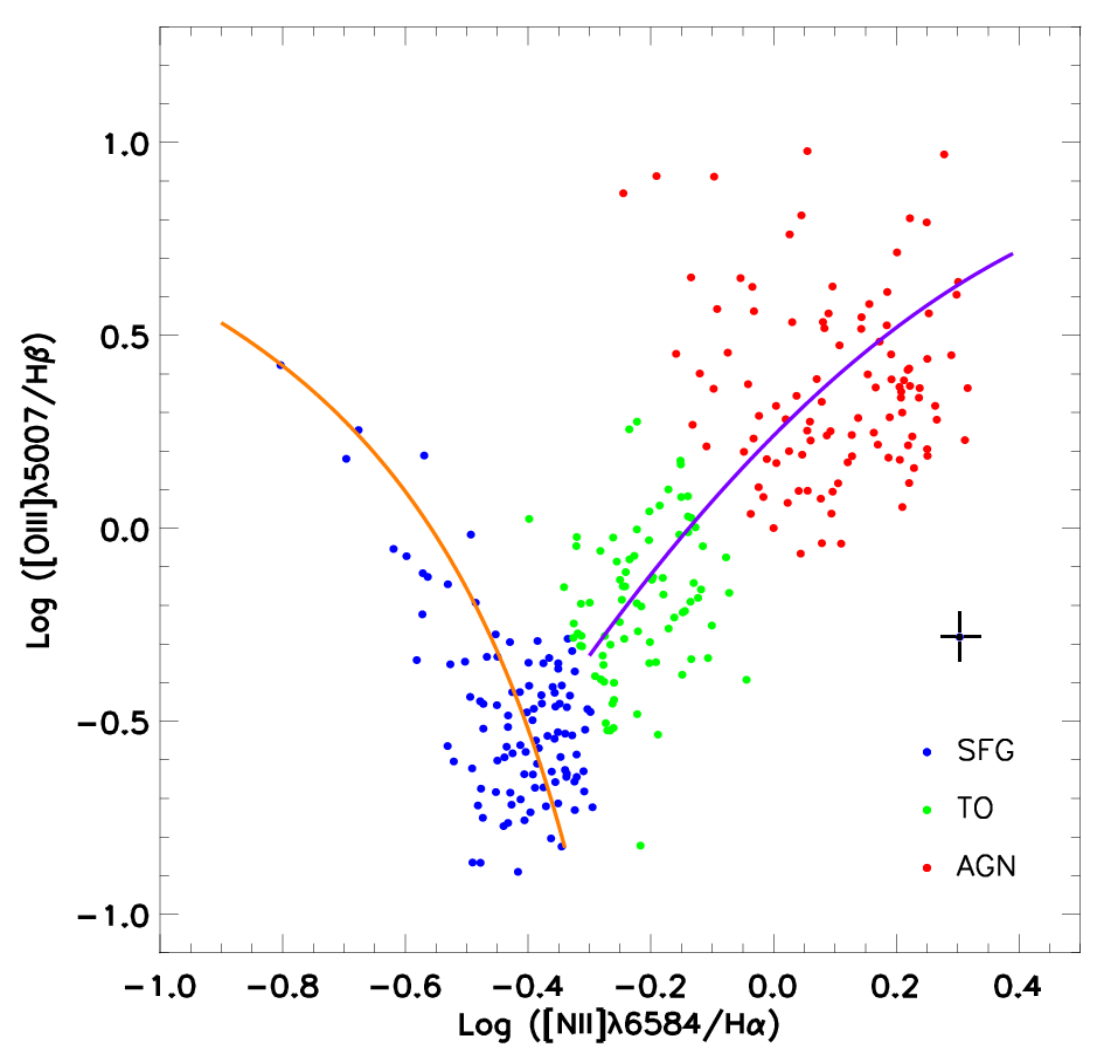

Figure 1: Metallicity calibrated diagnostic diagram. The inversion of the relation between $[\mathrm{O} / \mathrm{H}]$ and $[\mathrm{NII}] / \mathrm{H} \alpha$ for the AGNs explains the $v$ feature of the standard diagnostic diagram.

The activity types of the galaxies were determined using the standard diagnostic diagram, Figure 1, that compares the ratios [OIII] $\lambda 5007 / \mathrm{H} \beta$ and $[\mathrm{NII}] \lambda 6584 / \mathrm{H} \alpha$. The typical low uncertainty levels on these ratios (illustrated by the cross in Figure 1) have practically no effect on our classification. We distinguish 105 SFG galaxies (36.0\%), 83 TOs (28.4\%), and 104 AGNs (35.6\%). Note that we do not distinguish between S2 and LINERs in our sample, simply because of the lack of clear distinction in this diagnostic diagram between these two types of activity.

\section{Black Hole masses, luminosities and metallicities of the NLAGNs}

In two recent articles, Shemmer et al. [24] and Matsuoka et al. [19], it was shown that in BLAGNs the continuum luminosity at $5100 \AA$ and the black hole mass are correlated with the metallicity. The NLS1, however, seem to distinguish themselves by showing lower black hole masses for their high metallicities (see Figure 3b). However, if one transforms the luminosities into accretion rates, the NLS1 seem to follow the same relation as the BLAGNs (see Figure 4).

To verify if this behavior is also observed in the 104 NLAGNs in our sample, we have determined their BH masses, luminosities and estimated their metallicities [6]. The $\mathrm{BH}$ masses were obtained from the bulge masses, assuming the same relation as for the BLAGNs, proposed by Häring \& Rix [9]. The $\lambda L(5100 \AA)$ was measured directly in the SDSS spectra before subtracting the stellar population templates. We have verified that our method yields luminosities at $5100 \AA$ 


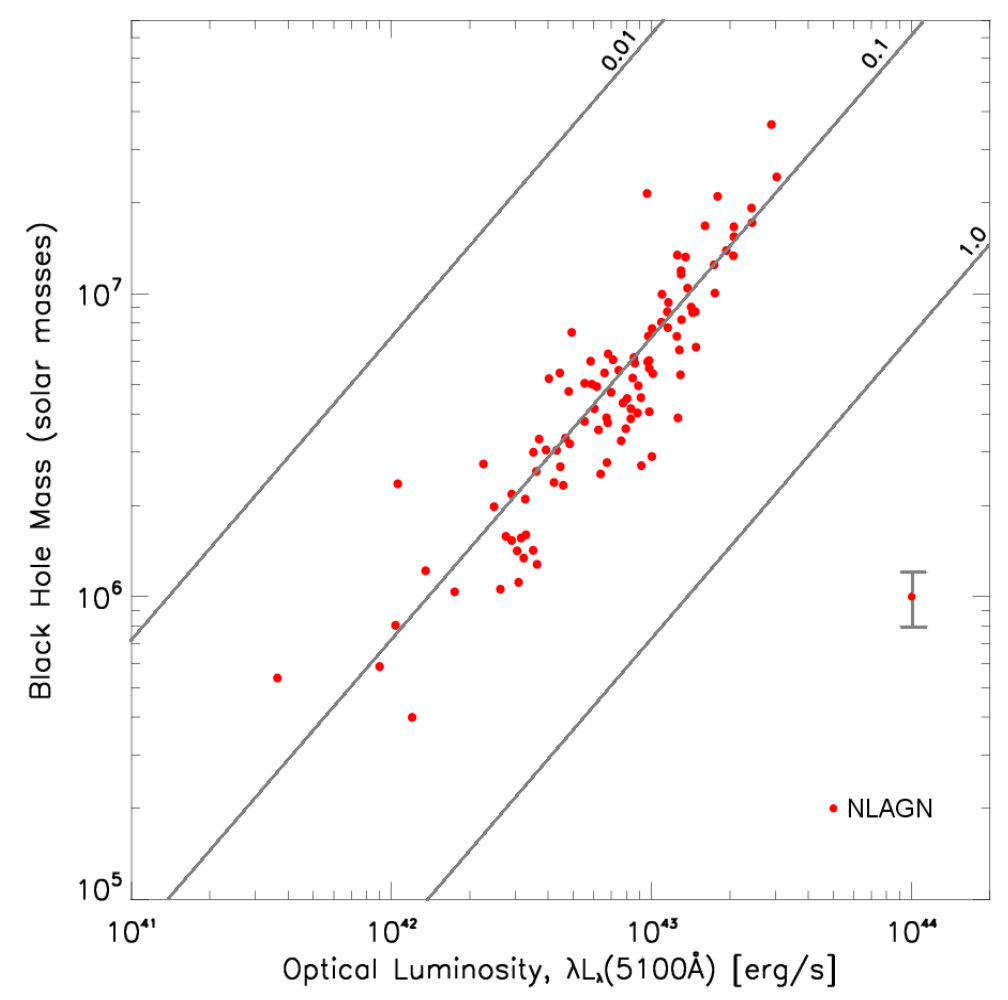

Figure 2: Black hole mass as a function of $5100 \AA$ luminosity for the NLAGNs. The typical uncertainty on the $\mathrm{BH}$ masses is indicated by a red dot with error bar in the lower right part of the figure.

Table 1: Pearson $(r)$ and Spearman $\left(r_{s}\right)$ correlation tests and chance probabilities.

\begin{tabular}{lcccc}
\hline$[\mathrm{O} / \mathrm{H}]$ versus & $r$ & $P(r)$ & $r_{s}$ & $P\left(r_{s}\right)$ \\
\hline$\lambda L_{\lambda}(5100)$ & 0.28 & 0.0037 & 0.28 & 0.0045 \\
$\mathrm{BH}$ mass & 0.28 & 0.0035 & 0.30 & 0.0028 \\
$L_{b o l} / L_{E d d}$ & -0.05 & 0.5932 & -0.09 & 0.3511 \\
\hline
\end{tabular}

similar (one-to-one relation) to those obtained by fitting a power law directly on this region of the spectra [7]. In Figure 2 we compare the BH masses with the luminosity $\lambda L(5100 \AA)$. The NLAGNs have BH masses much below $10^{8} \mathrm{M}_{\odot}$, which is the lower limit in BLAGNs. These masses are comparable to those measured in the NLS1 (Figure 3b).

The metallicity, $[\mathrm{O} / \mathrm{H}]$, was determined empirically by fitting-purple line in Figure 1-a correlation between $\mathrm{OIII}] \lambda 5007 / \mathrm{H}_{\beta}$ and $[\mathrm{NII}] / \mathrm{H} \alpha$ on the TOs, extrapolating over the AGNs, and by searching for a metallicity calibration that reproduces the values obtained by Bennert et al. [3] using CLOUDY. In Coziol et al. [6] we found that the calibration based on $R_{3}=1.35 \times\left([\mathrm{OIII}] \lambda 5007 / \mathrm{H}_{\beta}\right)$ as determined empirically for the SFGs yields a relatively good first approximation, with uncertainty of \pm 0.2 dex, but slightly higher for the S2 which are extreme objects compared to most of the AGNs in our sample. In Figure 1 the metallicity calibration for the NLAGNs region implies the ratio $[\mathrm{NII}] / \mathrm{H} \alpha$ increases as $[\mathrm{O} / \mathrm{H}]$ decreases through the relation: $[\mathrm{O} / \mathrm{H}]=-0.52+(\log ([\mathrm{NII}] / \mathrm{H} \alpha)$ $-0.6)^{2}$. This is consistent with the possibility of an overabundance of nitrogen in AGNs ([22]; 
[26]; [25]; [8]; [3]).

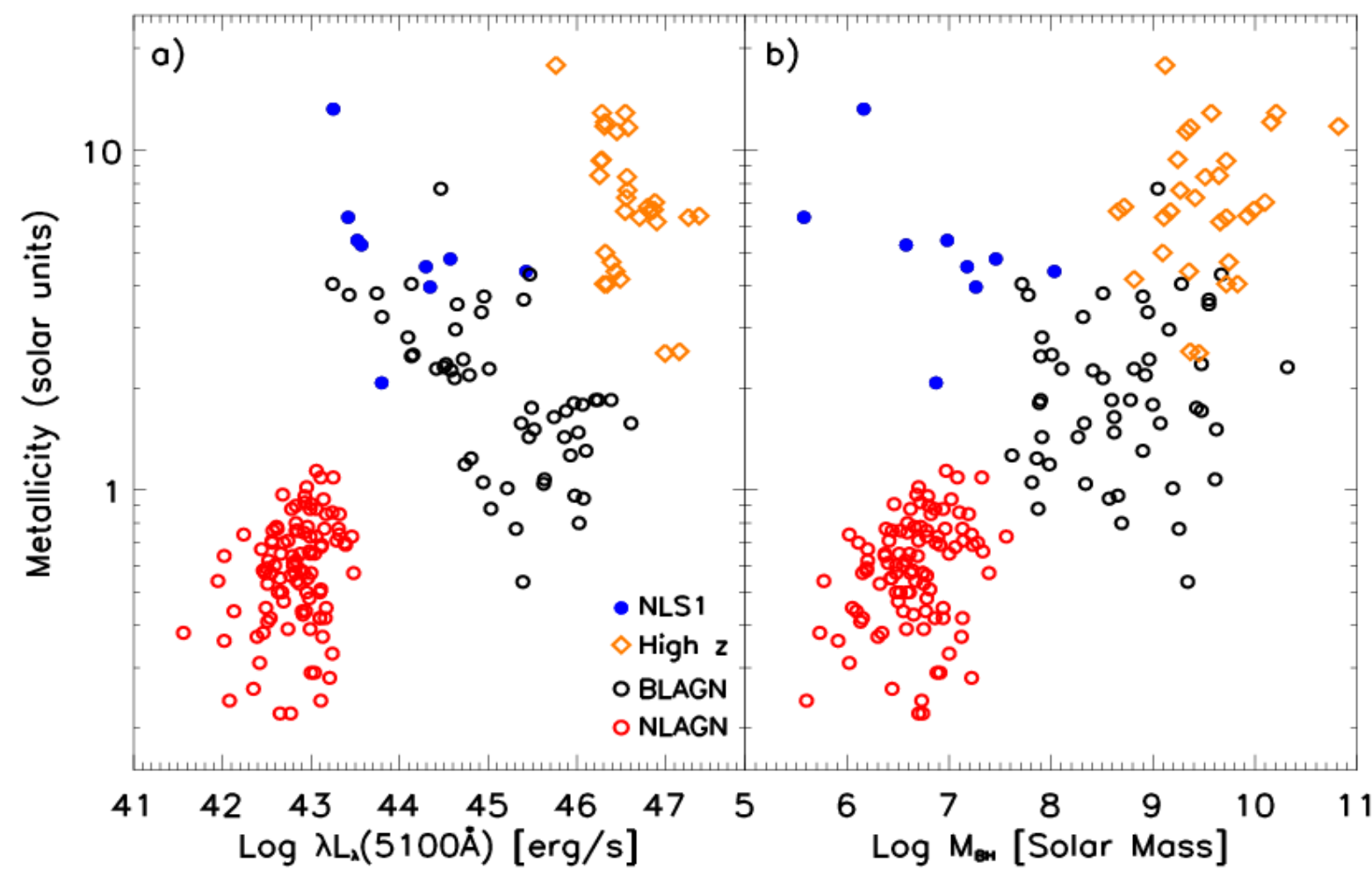

Figure 3: a) Metallicities in solar units, $\mathrm{Z} / \mathrm{Z}_{\odot}$, versus $5100 \AA$ luminosities, and b) versus Black hole masses.

In Figure 3 we compare the metallicities of the NLAGNs with their luminosities $\lambda L(5100 \AA)$ and $\mathrm{BH}$ masses. We find two statistically significant positive correlations, for $[\mathrm{O} / \mathrm{H}]$ with $\lambda L(5100 \AA)$ and $[\mathrm{O} / \mathrm{H}]$ with the $\mathrm{BH}$ mass (see Table 1 ).

In Figure 3 we also compare the NLAGNs with the BLAGNs at low and high $\mathrm{z}$ and the NLS1, as studied before by Shemmer et al. [24]. The transformation from Nv/Civ to [O/H] is based on the results of the model published by Hamann \& Ferland [8]. We have deduced the relation $Z_{\odot}=$ $0.126+8.859 \cdot(\mathrm{Nv} / \mathrm{CIV})-0.949 \cdot(\mathrm{Nv} / \mathrm{CIV})^{2}$. Note how well the NLAGNs in our sample seem to extend the relationships found for the BLAGNs towards the lower regime in $[\mathrm{O} / \mathrm{H}]$.

\section{The relation between metallicities and accretion rates}

To determine the bolometric luminosity, $L_{b o l}$, of the NLAGNs, we used the relation $L_{b o l}=$ $9 \times \lambda L(5100 \AA)$ [11]. Although our sample of NLAGNs considered alone shows no correlation between $[\mathrm{O} / \mathrm{H}]$ and the accretion rate $L_{b o l} / L_{E d d}$ (Table 1), added to the sample of Shemmer et al. [24], Figure 4, they seem to extend the relation found for the BLAGNs to lower metallicities.

In their analysis, Shemmer et al. [24] also found the NLS1 to have accretion rates comparable to BLAGNs, despite their lower BH masses, but consistent with their high metallicities. In Figure 4 the NLAGNs which have lower metallicities than the NLS1 accrete at lower rates, which is consistent with the metallicity-accretion rate relationship found by Shemmer et al. [24]. 


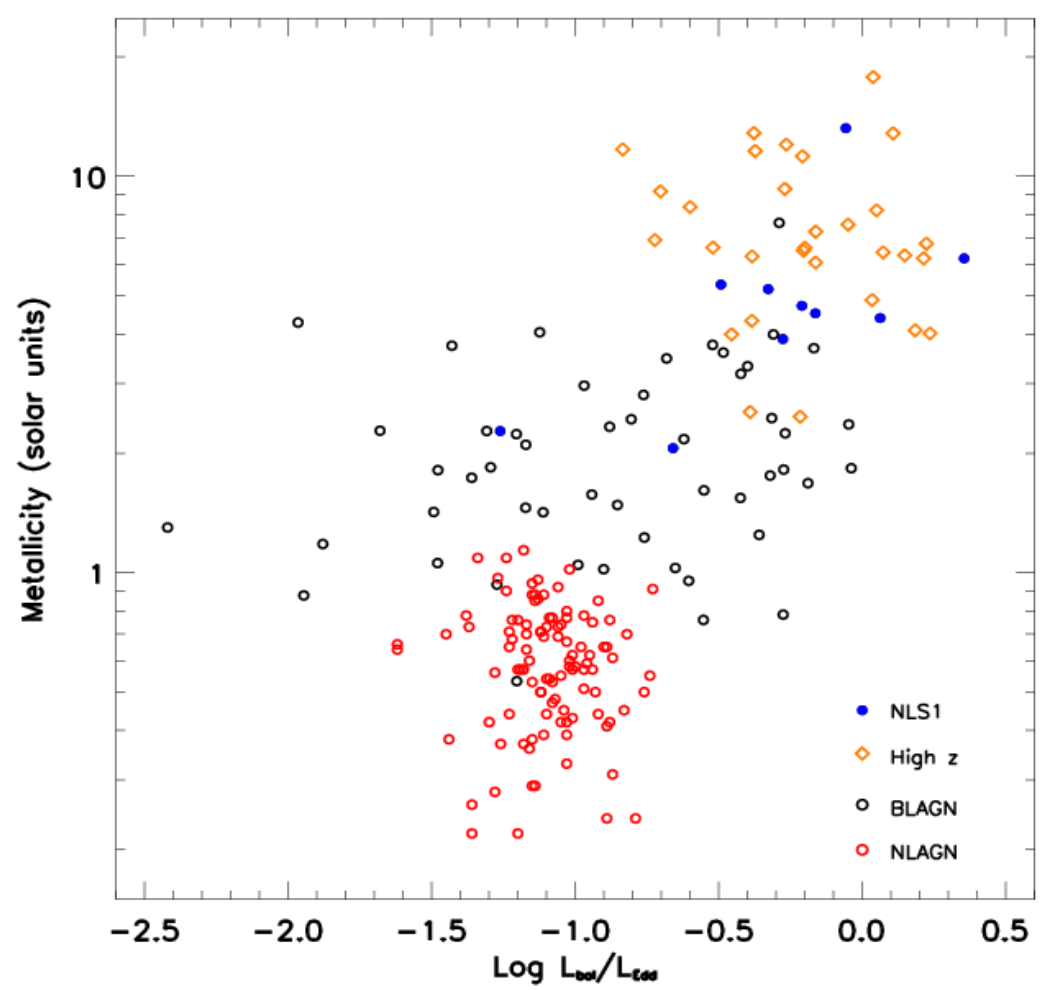

Figure 4: Metallicities in solar units versus accretion rates, $L_{b o l} / L_{E d d}$.

\section{Conclusions}

Based on our study, we formulate four conclusions:

1. The NLAGNs composed of S2 and LINERs are genuine AGNs. They simply have smaller mass BHs than the BLAGNs. They also show lower accretion rates, typically accreting matter at 0.1 the Eddington limit.

2. The metallicities of the NLAGNs are subsolar, varying between 0.3 and $1 Z_{\odot}$. Similarly to the BLAGNs, the NLAGNs show statistically significant positive correlations for the metallicity with the BH mass and the luminosity at $5100 \AA$.

3. The correlations observed for the NLAGNs seem to extend the correlations of the BLAGNs to the lower metallicity regime ([24]; [19]). In particular, we find a strong correlation between the black hole mass and metallicity, suggesting a common behavior for AGNs ([8]; [19]; [6]).

4. The NLAGNs is consistent with the relationship between the metallicity and accretion rate as previously discovered by Shemmer et al. [24] for the BLAGNs. The NLAGNs have BH masses similar to those of NLS1, but because their metallicities are lower, they accrete at lower rates. 


\section{Acknowledgments}

J.P. Torres-Papaqui acknowledges PROMEP for support grants 103.5-10-4684. I. PlauchuFrayn acknowledges postdoctoral fellowship No. 145727 from CONACyT, México. The authors would also like to thank N. Bennert and S. Komossa for making their results of CLOUDY models available to us, and O. Shemmer for furnishing the data for the BLAGNs.

\section{References}

[1] K.N. Abazajian, J.K. Adelman-McCarthy, M.A. Agüeros, and 201 coauthors, 2009, The Seventh Data Release of the Sloan Digital Sky Survey, ApJS, 182, 543.

[2] J.A. Baldwin, M.M. Phillips, \& R. Terlevich, 1981, Classification parameters for the emission-line spectra of extragalactic objects, PASP, $\mathbf{9 3}, 5$.

[3] N. Bennert, B. Jungwiert, S. Komossa, M. Haas, \& R. Chini, 2006, Size and properties of the NLR in the Seyfert-2 galaxy NGC 1386, A\&A, 446, 919.

[4] R. Cid Fernandes, A. Mateus, L. Sodré, G. Stasínska, \& J.M. Gomes, 2005, Semi-empirical analysis of Sloan Digital Sky Survey galaxies - I. Spectral synthesis method, MNRAS, 358, 363.

[5] R. Coziol, 1996, The history of star formation of starburst galaxies, A\&A, 309, 345.

[6] R. Coziol, J.P. Torres-Papaqui, I. Plauchu-Frayn, J.M. Islas-Islas, R.A. Ortega-Minakata, D.M. Neri-Larios, \& H. Andernach, 2011, The nature and origin of narrow-line AGN activity in galaxies, MNRAS, submitted.

[7] P.J. Francis, P.C. Hewett, C.B. Foltz, F.H. Chaffee, R.J. Weymann, S.L. Morris, 1991, A high signal-to-noise ratio composite quasar spectrum, ApJ, 373, 465.

[8] F. Hamann, \& G. Ferland, 1993, The Chemical Evolution of QSOs and the Implications for Cosmology and Galaxy Formation, ApJ, 418, 11.

[9] N. Häring \& H.W. Rix, 2004, On the Black Hole Mass-Bulge Mass Relation, ApJ, 604, L89.

[10] T.M. Heckman, 1980, An optical and radio survey of the nuclei of bright galaxies - Activity in normal galactic nuclei, $A \& A, \mathbf{8 7}, 152$.

[11] V.E. Karachentseva, S.N. Mitronova, O.V. Melnyk \& I.D. Karachentsev, 2010, Catalog of isolated galaxies selected from the 2MASS survey, AstBu, $\mathbf{6 5}, 1$.

[12] S., Kaspi, P.S., Smith, H., Netzer, D., Maoz, B.T., Jannuzi, U., Giveon, 2000, Reverberation Measurements for 17 Quasars and the Size-Mass-Luminosity Relations in Active Galactic Nuclei, ApJ, 533, 631.

[13] G. Kauffmann, T.M. Heckman, C. Tremonti, J. Brinchmann, S. Charlot, S.D.M. White, S.E. Ridgway, J. Brinkmann, M. Fukugita, P.B. Hall, Z. Ivezić, G.T. Richards, \& D.P. Schneider, 2003, The host galaxies of active galactic nuclei, MNRAS, 346, 1055.

[14] L.J. Kewley, B. Groves, G. Kauffmann \& T. Heckman, 2006, The host galaxies and classification of active galactic nuclei, MNRAS, 372, 961.

[15] L.J. Kewley, M.A. Dopita, R.S. Sutherland, C.A. Heisler \& J. Trevena, 2001, Theoretical Modeling of Starburst Galaxies, ApJ, 556, 121.

[16] S. Komossa \& D. Xu, 2007, Narrow-line seyfert 1 galaxies and the $M_{B H^{-}} \sigma$ relation, ApJ, 667, L33. 
[17] S. B. Kraemer, I. M. George, D. M. Crenshaw \& J. R. Gabel, 2004, On the relationship between the optical emission-line and $x$-ray luminosities in seyfert 1 galaxies, ApJ, 607, 794.

[18] J.H. Krolik, in “Active Galactic Nuclei”, Princeton University Press, 1999.

[19] K. Matsuoka, T. Nagao, A. Marconi, R. Maiolino, \& Y. Tanigushi, 2011, The mass-metallicity relation of SDSS quasars, $A \& A, \mathbf{5 2 7}, 100$.

[20] C.J. Miller, R.C. Nichol, P.L. Gómez, A.M. Hopkins, \& M. Bernardi, 2003, The Environment of Active Galactic Nuclei in the Sloan Digital Sky Survey, ApJ, 597, 142.

[21] D.E. Osterbrock, 1989, Astrophysics of Gaseous Nebulae and Active Galactic Nuclei, University Science Books.

[22] D.E. Osterbrock, 1970, Abundances of the Elements in Caseous Nebulae. QJRAS, 11, 199.

[23] K. A. Pounds, C. Done, \& J. P. Osborne, 1995, RE 1034+39: a high-state Seyfert galaxy?, MNRAS, 277, L5.

[24] O. Shemmer, H. Netzer, R. Maiolino, E. Oliva, S. Croom, E. Corbett, \& L. di Fabrizzio, 2004, Near-Infrared Spectroscopy of High-Redshift Active Galactic Nuclei. I. A Metallicity-Accretion Rate Relationship, ApJ, 614, 547.

[25] T. Storchi Bergmann, 1991, On the ratio (N II)/H-alpha in the nucleus of Seyfert 2 and LINER galaxies. MNRAS, 249, 404.

[26] T. Storchi Bergmann, \& M.G. Pastoriza, 1989, On the metal abundance of low-activity galactic nuclei. ApJ, 347, 195.

[27] J.P. Torres-Papaqui, R. Coziol, H. Andernach, J.M. Islas-Islas, R.A. Ortega-Minakata, D.M. Neri-Larios, \& I. Plauchu-Frayn, 2011, LLAGNs in dense galactic environments: evidence of dying quasars in massive early type galaxies, MNRAS, submitted.

[28] S. Veilleux, \& D.E. Osterbrock, 1987, Spectral classification of emission-line galaxies, ApJS, 63, 295.

[29] D.W. Weedman, 1986, Quasar Astronomy, Cambridge University Press. 